\title{
Growth and Collapse Dynamics of a Vapor Bubble near or at a Wall
}

\author{
Huigang Wang ${ }^{1}$, Chengyu Zhang ${ }^{2}$ and Hongbing Xiong ${ }^{1, * \mathbb{D}}$ \\ 1 Key Laboratory of Soft Machines and Smart Devices of Zhejiang Province, Zhejiang University, \\ Hangzhou 310027, China; 21824075@zju.edu.cn \\ 2 Geely Automobile Research Institute, Ningbo 315336, China; cyu.zhang@geely.com \\ * Correspondence: hbxiong@zju.edu.cn
}

check for updates

Citation: Wang, H.; Zhang, C.; Xiong, H. Growth and Collapse Dynamics of a Vapor Bubble near or at a Wall. Water 2021, 13, 12. https:/ /dx.doi.org/ 10.3390/w13010012

Received: 4 November 2020 Accepted: 17 December 2020 Published: 24 December 2020

Publisher's Note: MDPI stays neutral with regard to jurisdictional claims in published maps and institutional affiliations.

Copyright: () 2020 by the authors. Licensee MDPI, Basel, Switzerland. This article is an open access article distributed under the terms and conditions of the Creative Commons Attribution (CC BY) license (https: / creativecommons.org/ licenses/by/4.0/).

\begin{abstract}
This study investigated the dynamics of vapor bubble growth and collapse for a laserinduced bubble. The smoothed particle hydrodynamics (SPH) method was utilized, considering the liquid and vapor phases as the van der Waals (VDW) fluid and the solid wall as a boundary. We compared our numerical results with analytical solutions of bubble density distribution and radius curve slope near a wall and the experimental bubble shape at a wall, which all obtained a fairly good agreement. After validation, nine cases with varying heating distances (L2 to L4) or liquid heights (h2 to h10) were simulated to reproduce bubbles near or at a wall. Average bubble radius, density, vapor mass, velocity, pressure, and temperature during growth and collapse were tracked. A new recognition method based on bubble density was recommended to distinguish the three substages of bubble growth: (a) inertia-controlled, (b) transition, and (c) thermally controlled. A new precollapse substage (Stage (d)) was revealed between the three growth stages and collapse stage (Stage (e)). These five stages were explained from the out-sync between the bubble radius change rate and vapor mass change rate. Further discussions focused on the occurrence of secondary bubbles, shockwave impact on the wall, system entropy change, and energy conversion. The main differences between bubbles near and at the wall were finally concluded.
\end{abstract}

Keywords: cavitation; boiling; nanobubble; plasmonic bubble; phase change

\section{Introduction}

Vapor bubbles have recently drawn intensive attention in many research fields [1], such as micro- or nanomanipulation [2], the heat transfer of two-phase heat exchangers [3,4], and medical vapor bubble cancer treatment [5-7]. However, vapor bubble dynamics are incredibly complex and nonlinear [8-10], involving bubble oscillations and interface fluctuations during growth, shockwave impact, and cavitation noise during collapse. Understanding bubble growth and collapse mechanisms is key to successfully solving the challenges connected to these applications.

Many studies have focused on vapor bubble dynamics, both experimentally or numerically. With regard to experiments, pulsed lasers are usually used to produce vapor bubbles, and high-speed cameras observe bubble dynamics under the bubble-free surface or bubble-solid wall interactions [11,12]. Gonzalez-Avila et al. studied the dynamics of bubbles in a highly variable liquid gap [13], and Sun and Zachary et al. concluded that thermal effects played an important role in the entire growth and collapse of bubbles in microchannels $[14,15]$. Kangude et al. explained the growth mechanism of vapor bubbles on hydrophobic surfaces with the help of infrared thermal imaging measurement methods [16].

In addition to experiments, numerical simulations could help to better understand bubble dynamics and provide more details about bubble density, velocity, and heat fluctuations, including volume of fraction (VOF), the lattice Boltzmann method (LBM), and smoothed particle hydrodynamics (SPH). Among them, volume of fraction (VOF) is 
one of the conventional numerical tools based on the finite-volume method, and Rayleigh and Plesset $[9,17]$ expanded on the bubble growth theory. According to Rayleigh, the early inertia growth stage of a bubble is affected by the pressure difference between a bubble and the surrounding liquid [18], whereas Plesset believes that vapor growth is partly caused by the evaporation of superheated liquid $[19,20]$. Prosperetti characterized the dynamics of bubble growth into three stages: (a) the inertia-controlled stage, (b) the transition stage, (c) and the thermally controlled stage (involving vapor bubbles). Although these analytical models provide insights into bubble growth, they still need a supplementary model at the liquid-vapor interface to account for the thermal effect and bubble collapse. For example, Liang developed a buoyancy and pressure-difference model to study the thermal growth of bubbles in superheated liquid [21].

When a bubble grows beyond its limits, it begins to collapse due to the pressure of its surrounding liquid. Pavlov used a model to calculate the temperature and pressure at bubble collapse, indicating that there were severe thermal changes and pressure shocks in the final stage of collapse [22]. Qin's numerical results demonstrated that when a bubble collapses, it outputs a large amount of conduction heat to its surroundings [23]. Zhang studied the collapse of a group of bubbles and noted that potential energy was transformed into wave mechanical energy after collapse [24].

Most of the above-mentioned studies are limited either in bubble growth or bubble collapse, providing many assumptions. Only a few works have completely covered the whole stage of growth and collapse. For example, Wang built a model to calculate vapor bubble growth and collapse in microgravity but used a lot of empirical parameters and formulas for determining the bubble interface [25]. These works could not provide thorough information from bubble growth to bubble collapse, as bubble growth would affect the subsequent collapse. Thus, it is necessary to establish a full-field model able to cover liquid and vapor phases with their phase change.

The lattice Boltzmann method (LBM) is based on the Boltzmann equation, using the lattice field to solve the mass, momentum, and energy transport process. Chen et al. constructed a multiphase LBM flow model to study the process of the growth and departure of bubbles [26]. Yang et al. investigated the thermodynamics of a collapsing cavitation bubble, analyzing the maximum temperature, velocity, and pressure field of a bubble near a wall [27].

Different from the above sharp interface methods, the diffusion interface method (DIM) treats the vapor and liquid phase interface as continuous. There is no additional modeling of the interface, which could provide a complete hydrodynamic description of bubble growth and collapse. Wang used the DIM to capture the bubble interface to study the growth of vapor bubbles near a wall [28]. Gallo used the fluctuating hydrodynamic theory and DIM to build a vapor bubble model from bubble growth to bubble oscillation [29].

Our method is based on the DIM, using the SPH numerical algorithm to solve the Navier-Stoke-Korteweg (NSK) equations for the growth and collapse of vapor bubbles. Section 2 describes the details of this method. In Section 3, we validate our model with the analytical solution provided by Felderhof $[29,30]$ and bubble growth rate from Lee and Merte [10,17], where good agreements were obtained. Section 4 shows our numerical results and discussion. Section 4.1 presents the numerical setup. Section 4.2 focuses on bubble growth near a wall. Section 4.3 examines bubble growth and collapse at a wall. Section 5 presents our conclusions.

\section{SPH Modeling}

In our model, compressible vapor and liquid are considered a two-phase fluid with continuous density gradients. In the Lagrange formula, the liquid and gas phase uniformly follows the conservation equations of mass, momentum, and energy:

$$
\frac{d \rho}{d t}=-\rho \nabla \cdot \mathbf{v}
$$




$$
\begin{gathered}
\frac{d \mathbf{v}}{d t}=\frac{1}{\rho} \nabla \cdot \mathbf{M} \\
\frac{d U}{d t}=\frac{1}{\rho} \mathbf{M}: \nabla \mathbf{v}+\frac{\kappa}{\rho} \nabla^{2} T
\end{gathered}
$$

where $\rho$ is the density, $\mathbf{v}$ is the velocity vector, $\mathbf{M}$ is the stress tensor, $T$ is the temperature, $U$ is the internal energy, and $\kappa$ is the thermal conductivity. The stress tensor $\mathbf{M}$ includes pressure terms, shear and bulk viscosity terms, as well as an additional Korteweg tensor term $\mathbf{M} c$ of the gas-liquid diffusion interface, as shown below:

$$
\mathbf{M}=-p \mathbf{I}+\eta_{s}\left(\nabla \mathbf{v}+\nabla \mathbf{v}^{T}\right)+\left(\eta_{v}-\frac{2}{\operatorname{dim}} \eta_{s}\right)(\nabla \cdot \mathbf{v}) \mathbf{I}+\mathbf{M}_{C}
$$

where $p$ represents pressure, dim represents the dimension of space, and $\eta_{s}$ and $\eta_{v}$ are shear and volume viscosity, respectively. The Korteweg tensor Mc can be used to simulate the capillary force on the interface due to the density gradient, expressed as:

$$
\mathbf{M}_{C}=K\left(\rho \nabla^{2} \rho+\frac{1}{2}|\nabla \rho|^{2}\right) \mathbf{I}-K \nabla \rho \nabla \rho
$$

where $K$ is the gradient energy coefficient.

In order to close the momentum and energy equations, the VDW equation is chosen to describe the pressure state equation, which can describe the gas-liquid coexistence system. The expression of the van der Waals equation of state is:

$$
p=\frac{\rho \bar{k}_{b} T}{1-\bar{\beta} \rho}-\bar{\alpha} \rho^{2}
$$

where $\bar{k}_{b}$ is the Boltzmann constant, $\bar{\alpha}$ is the parameter of attraction, and $\bar{\beta}$ is related to the size of the particles. $\bar{k}_{b}, \bar{\alpha}$, and $\bar{\beta}$ are set as 1,2 , and 0.5 for the van de Waals fluid, respectively.

Considering the thermodynamic relationship of the system, the thermodynamic consistency formula could be expressed as [31]:

$$
T d S=d U+P d V
$$

and the total differential form of entropy $S(T, V)$ is:

$$
d S=\left(\frac{\partial S}{\partial V}\right)_{T} d T+\left(\frac{\partial S}{\partial T}\right)_{V} d V
$$

From Maxwell's relationship $\left(\frac{\partial S}{\partial V}\right)_{T}=\left(\frac{\partial P}{\partial T}\right)_{V^{\prime}}$ constant volume specific heat capacity $C_{V}=T\left(\frac{\partial S}{\partial T}\right)_{V}$ and the van der Waals equation of state Equation (6), we can obtain the total differential form of the internal energy $d U$ :

$$
d U=C_{V} d T+\frac{\bar{\alpha}}{V^{2}} d V
$$

Integrating Equation (9) and substituting $C_{V}=\operatorname{dim} \frac{\bar{k}_{b}}{2}, U(T, V)$ can be expressed by $U(T, \rho)$ :

$$
U=\frac{\operatorname{dim}}{2} \bar{k}_{b} T-\bar{\alpha} \rho
$$

The former term $\frac{\operatorname{dim}}{2} \bar{k}_{b}$ is defined as thermal energy, and the latter term $-\bar{\alpha} \rho$ is defined as potential energy. 
For the closed governing equations of Equations (1)-(3), the SPH method is used to discretize them into numerical forms. Equation (11) is used to calculate the mass equation with second-order accuracy:

$$
\rho_{a}=\frac{\sum_{b} m_{b} W_{a b}}{\sum_{b} m_{b} / \rho_{b} W_{a b}}
$$

where $m$ is the particle mass, the subscript $b$ indicates the adjacent particle around the particle $a$, and $W_{a b}$ is a kernel function, which explains the particle distance between particles $a$ and $b$. Here, the vapor or liquid phase is determined through the critical fluid density of the VDW fluid. If the fluid density is less than the critical density, then it is the vapor phase; otherwise, it is the liquid phase.

In the VDW fluid, the momentum and energy equations should be divided into short-range and long-range because this treatment can accurately deal with the surface tension effect. Thus, the momentum and energy numerical equations could be represented as:

$$
\begin{gathered}
\frac{d v_{a}}{d t}=\sum_{b} m_{b}\left(\frac{\mathbf{M}_{a}}{\rho_{a}^{2}}+\frac{\mathbf{M}_{b}}{\rho_{b}^{2}}\right) \cdot \nabla W_{a b}+\sum_{b} m_{b}\left(\frac{\mathbf{M}_{a}^{H}}{\rho_{a}^{2}}+\frac{\mathbf{M}_{b}^{H}}{\rho_{b}^{2}}\right) \cdot \nabla W_{a b}^{H} \\
\frac{d U_{a}}{d t}=\frac{1}{2} \sum_{b} m_{b}\left(\frac{\mathbf{M}_{a}}{\rho_{a}^{2}}+\frac{\mathbf{M}_{b}}{\rho_{b}^{2}}\right): \mathbf{v}_{b a} \nabla W_{a b}+\frac{1}{2} \sum_{b} m_{b}\left(\frac{\mathbf{M}_{a}^{H}}{\rho_{a}^{2}}+\frac{\mathbf{M}_{b}^{H}}{\rho_{b}^{2}}\right): \mathbf{v}_{b a}^{H} \nabla W_{a b}^{H} \\
+\kappa \sum_{b} \frac{2 m_{b} \mathbf{r}_{a b} \cdot \nabla W_{a b}}{\rho_{a} \rho_{b}\left(\left|\mathbf{r}_{a b}\right|^{2}+0.01 h_{b}^{2}\right)} T_{a b}
\end{gathered}
$$

where the long-distance items are marked with superscript $H$, and the short-distance items are unmarked.

In this paper, we use the hyperbolic-shaped kernel function proposed by Yang [32]. The fluids of liquid and vapor are represented by continuous particles, compact or sparse, depending on their local density. Mirror ghost particles are used for the solid wall to ensure the no-slip boundary condition. Further information about this numerical model is provided in our previous work [33].

\section{Validation}

We firstly simulated the generation of a laser-induced spherical vapor bubble, which caused the outward expansion of fluid to produce a high-temperature vapor in the central region of the flow field. In Figure 1, we show the typical characteristics of a vapor bubble under laser heating. In Felderhof's study, the density distribution along the bubble radius is as follows [34]:

$$
\rho(\mathrm{r})=\frac{\rho_{L}+\rho_{V}}{2}-\frac{\rho_{L}-\rho_{V}}{2} \tanh \left(\frac{\mathrm{r}-R_{b}}{w}\right)
$$

where $\rho_{L}$ is the liquid density, $\rho_{V}$ is the vapor density, and $w$ is the width of the gas-liquid interface. Our model is based on the diffuse interface description of a two-phase liquid-vapor system endowed with thermal fluctuations. After bubble growth, we could observe that the inertial-driven bubble oscillates slightly in the confined systems. The average fluid density agrees well with Equation (14) in Figure 2. Gallo et al. investigated the nucleation of vapor bubbles in stretched or overheated liquids and found a similar phenomenon of bubble oscillation [29]. 


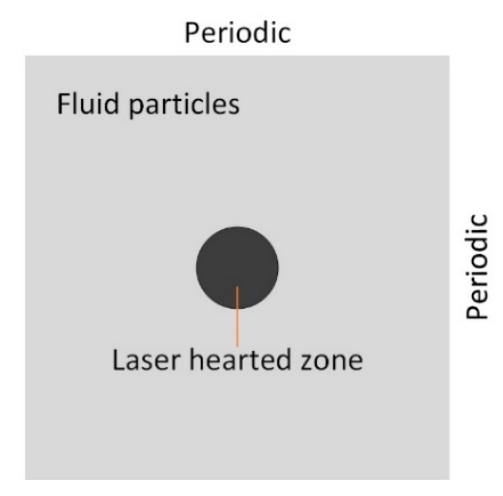

(a)

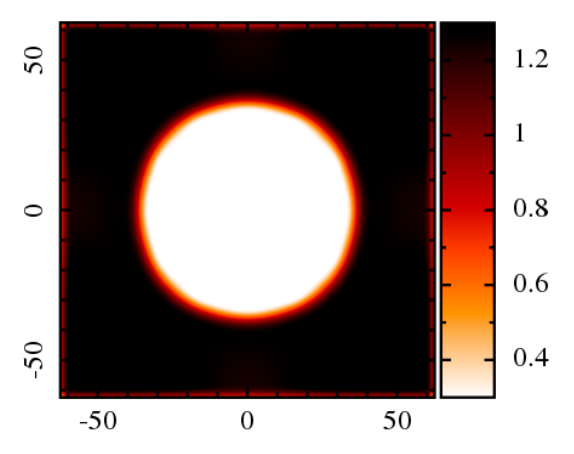

(b)

Figure 1. (a) Initial calculation domain; (b) snapshot of the vapor-filled laser-induced bubble.

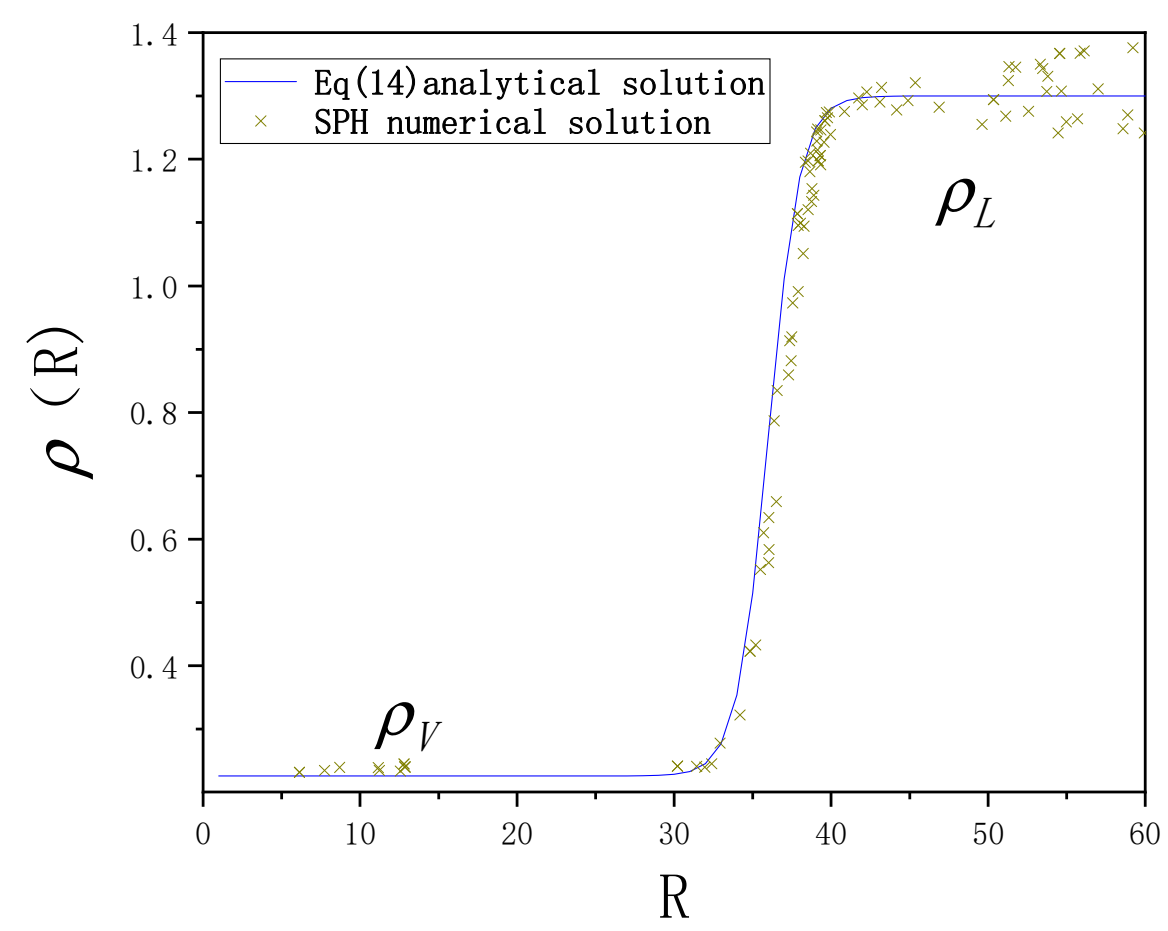

Figure 2. Radial density distribution of fluid across the bubble interface.

According to the curve slope of the bubble radius with time, three control mechanisms could be observed during bubble growth, categorized into the (a) inertia-controlled stage, (b) transition stage, and (c) thermally controlled stage. Lee and Merte [17] found that the curve slope in Stage (a) is twice as much as that in Stage (c) in logarithmic coordinates, as shown in Figure 3a. Our simulation obtained the logarithmic fitting coefficients for the radius to time as 2.2098 and 1.1043 in Stages (a) and (c), respectively, as shown in Figure $3 \mathrm{~b}$. This ratio is approximately $2: 1$, which is consistent with the analytical results of Lee and Merte. 


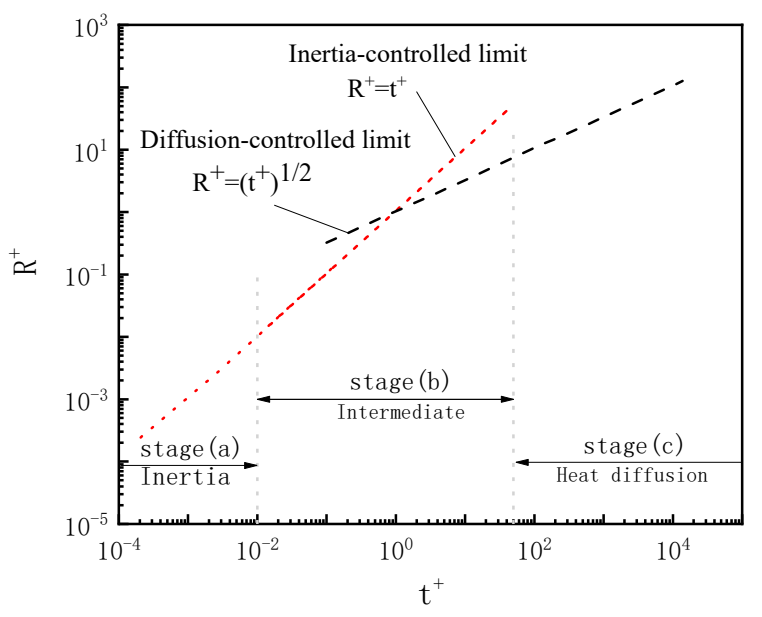

(a)

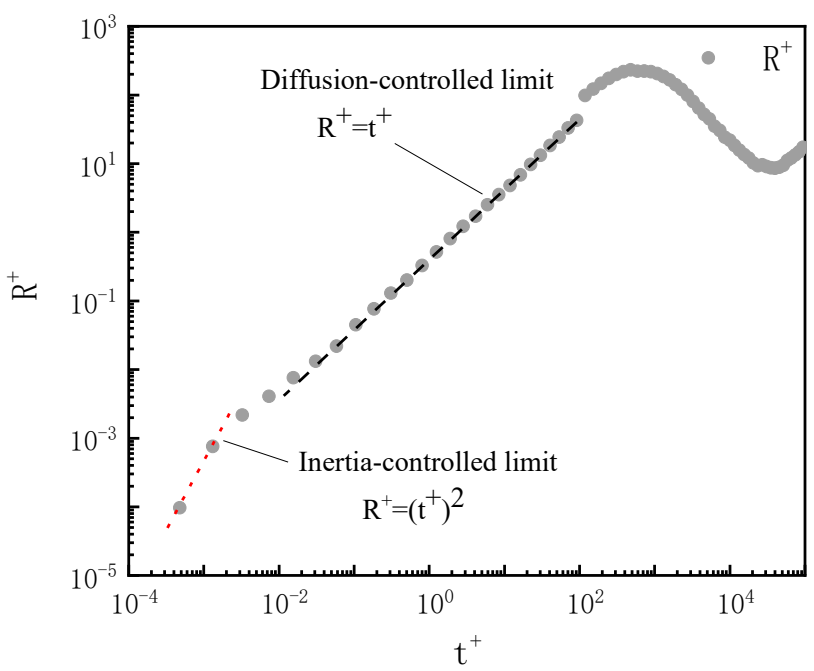

(b)

Figure 3. (a) Change of radius with time in stages of purely inertial growth and thermally controlled growth from Lee and Merte [17]; (b) numerical results of the smoothed particle hydrodynamics (SPH) of the radius-time curve at different stages of bubble growth.

\section{Result}

\subsection{Set-Up}

The SPH simulation started from the steady-state liquid, determined by the binodal line of the van der Waals fluid. The critical fluid density was introduced to distinguish between liquid and vapor, with $\rho_{c}=\frac{1}{3 \bar{b}}=\frac{2}{3}$. The SPH liquid particle mass $m=0.6$, steady density $\rho=1.2029$, and distance $d x=\sqrt{m / \rho}$ were initially arranged at a size of $400 \mathrm{dx} \times \mathrm{hdx}$ at the bottom, where $\mathrm{h}$ is the liquid height as shown in Figure 4 . We tried $500 \mathrm{dx}, 400 \mathrm{dx}, 300 \mathrm{dx}$, and $200 \mathrm{dx} \times$ hdx cases, respectively. Among these cases, the $400 \mathrm{dx} \times$ hdx case provided results that were good enough. The upper and bottom walls were treated as the stationary and insulated solid wall, and the left and right boundaries were the periodic boundaries. Gravity was considered in the downward direction. The whole chamber size was $400 \mathrm{dx} \times 200 \mathrm{dx}$. The heating area was within a radius of $12 \mathrm{dx}$ at the heating distance $\mathrm{L}$ above the wall to a superheat of $\Delta \mathrm{T}=10.33$. This region, heated by a laser, is spherical and homogeneous in this paper. We conducted a series of cases by changing the liquid height $\mathrm{h}$ and heating distance $\mathrm{L}$. The main characteristics of bubble growth and collapse in these cases could be classified by two deduced nondimensional 
parameters of heating distance and liquid height: $\gamma=\mathrm{L} \times \mathrm{dx} / \mathrm{Rx}, \eta=\mathrm{h} \times \mathrm{dx} / \mathrm{Rx}$. The key parameters of $L, h, \gamma, \eta, \eta-\gamma$, and $\mathbf{N}_{\text {secb }}$ are shown in Table 1 for each case. Here, $\eta-\gamma$ is related to the hydrostatic pressure, and $\mathbf{N}_{\text {secb }}$ is the number of secondary bubbles.

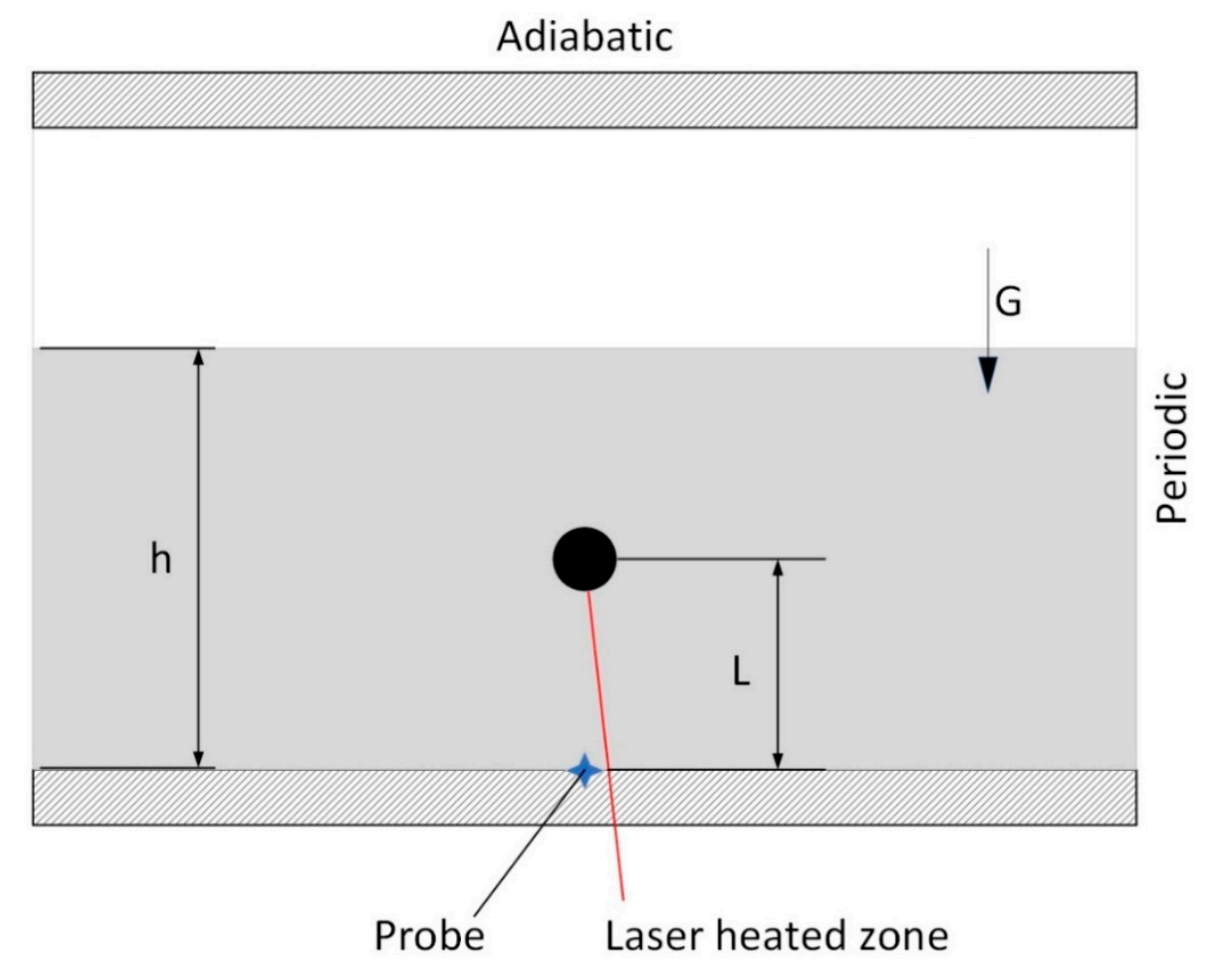

Figure 4. Computational domain.

Table 1. Case parameters.

\begin{tabular}{cccccccc}
\hline Type & CASE ID & $\mathbf{h} / \mathbf{d} \mathbf{x}$ & $\mathbf{L} / \mathbf{d} \mathbf{x}$ & $\boldsymbol{\gamma}=\mathbf{L} / \mathbf{R}_{\mathbf{x}}$ & $\boldsymbol{\eta}=\mathbf{h} / \mathbf{R}_{\mathbf{x}}$ & $\boldsymbol{\eta} \mathbf{\gamma}$ & $\mathbf{N}_{\text {secb }}$ \\
\hline \multirow{3}{*}{ Near the wall } & L2 & 100 & 20 & 1.0027 & 5.0128 & 4.0101 & 0 \\
& L3 & 100 & 30 & 1.3466 & 4.4887 & 3.1421 & 0 \\
& L4 & 100 & 40 & 1.0288 & 2.5721 & 1.5433 & 3 \\
\hline \multirow{5}{*}{ At the wall } & h10 & 100 & 0 & 0 & 5.7777 & 5.7777 & 0 \\
& h8 & 80 & 0 & 0 & 4.5021 & 4.5021 & 0 \\
& h7 & 70 & 0 & 0 & 2.5743 & 2.5743 & 1 \\
& h6 & 60 & 0 & 0 & 1.8387 & 1.8387 & 1 \\
& h4 & 40 & 0 & 0 & 0.8228 & 0.8228 & NA \\
& h2 & 20 & 0 & 0 & $<0.5$ & $<0.5$ & NA \\
\hline
\end{tabular}

\subsection{Bubble near the Wall}

Three cases, L2, L3 and L4, were examined for determining the bubble dynamics near the solid wall. Here, the liquid height $h$ was 100, and the heating distance $L$ varied from 20-30 to 40 regarding the initial liquid particle spacing distance dx. Figure 5 shows the representative bubble shapes at different moments for different cases. It was found that the bubble rose obviously during growth, and the spherical shape of the bubble was slightly distorted. We calculated the volume of the bubble and obtained the average radius of the bubble with time, as shown in Figure 6. It was found that during the entire bubble growth process, the change of heating distance $\gamma$ mainly affected the average radius of the bubble and the transition time from Stages (a) to (c) in the early growth stage. The greater the heating distance, the larger the bubble radius, and the later the transition time. 

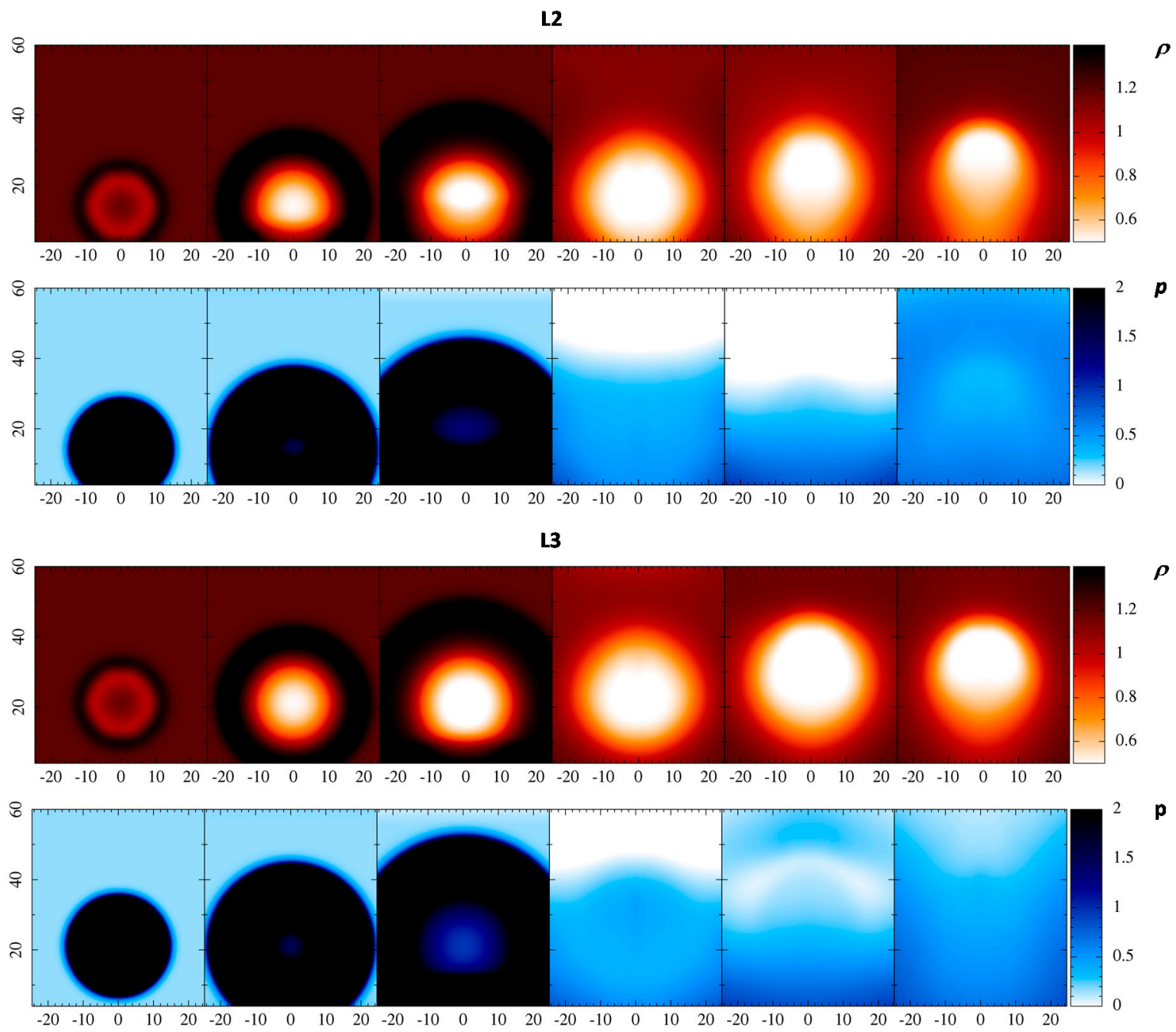

L4
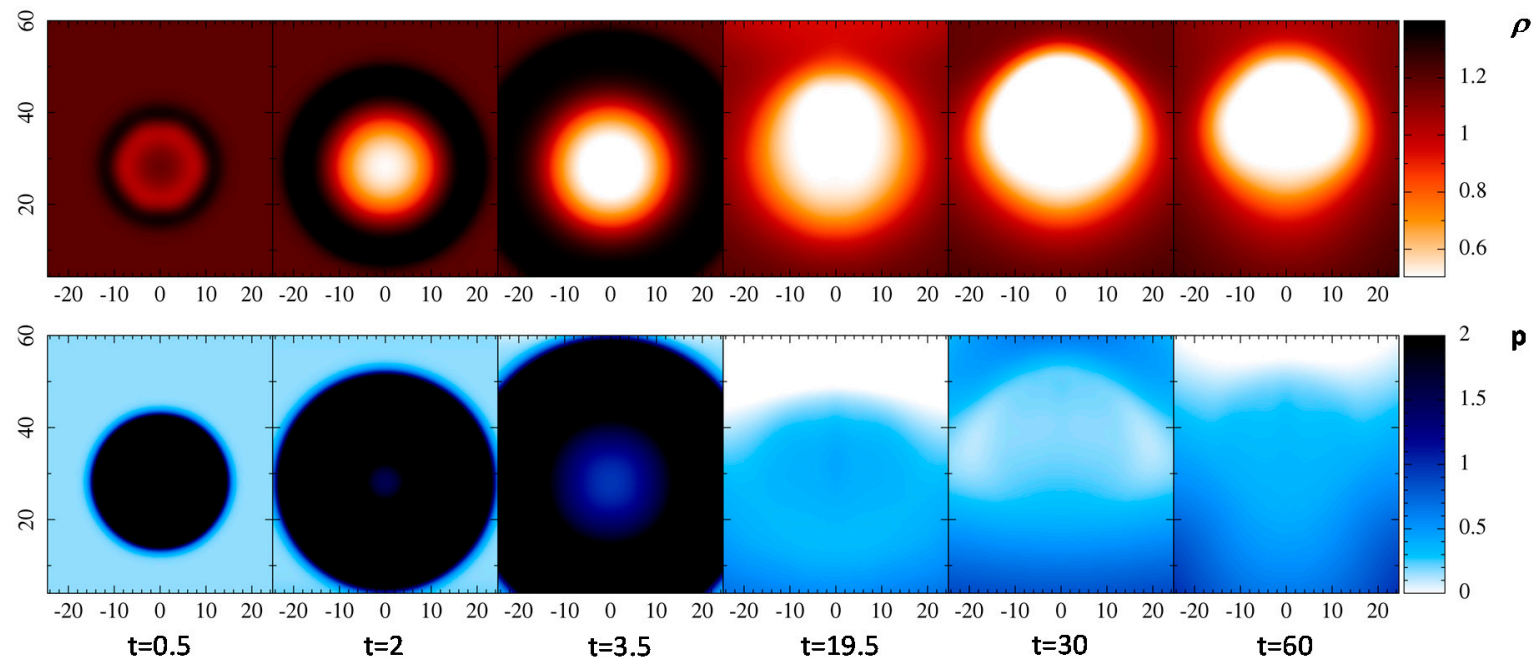

Figure 5. Density contour (top) and pressure contour (below) during bubble growth. 


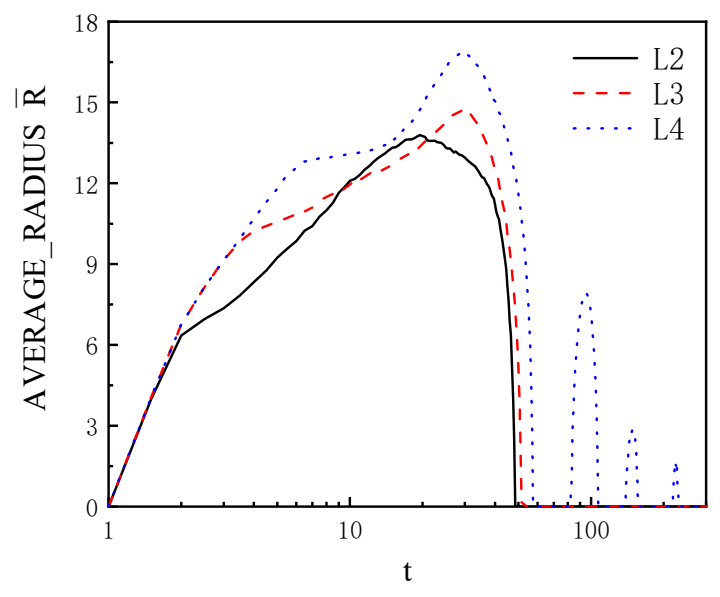

Figure 6. Average bubble radius $\overline{\mathrm{R}}$ with time.

In many studies, the vapor pressure, density, and temperature are considered constant, and the liquid is considered incompressible. Our study is different. The vapor, as well as the liquid, are compressible, thus we could capture the oscillation of bubble density during bubble growth and collapse, as shown in Figure 7.

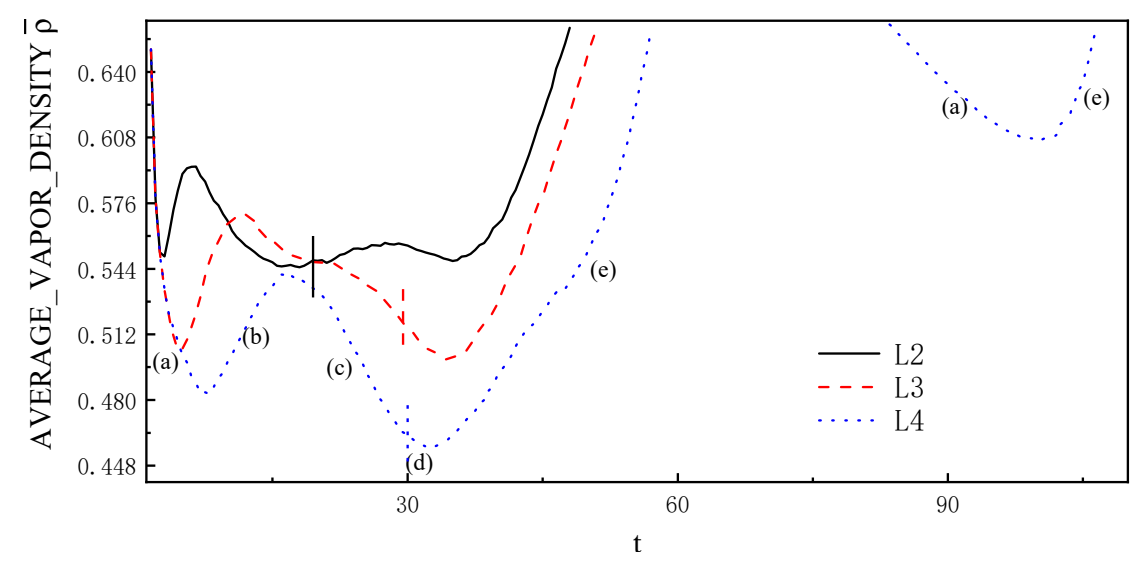

Figure 7. Average vapor density of the first bubble with time. Note that the vertical line is the moment when the bubble reaches maximum volume. Five bubble stages are concluded: (a) inertial controlled, (b) intermediate, (c) heat-diffused controlled, (d) precollapse, (e) collapse.

From Figure 7, we can clearly observe the transition time from Stages (a) to (b) as the first inflection point and the transition time from Stages (b) to (c) as the second inflection point. After the bubble radius reaches its maximum volume, there still exists a time gap before the bubble density increases continuously, which we call Stage (d). The precollaps of Stage (d) is from the point of the largest bubble radius to the next minimum value of bubble density. We define the last vapor inflection point to vanish as collapse (Stage (e)). The secondary bubble in Case L4 only has Stages (a) and (e), as shown by the inflection point in Figure 7. The reason is that the lifetime of the secondary bubble is too short. The bubble density profile could provide a clear and easy criterion between these five substages (Stages (a)-(e)). Therefore, the recognition method based on density is better for determining bubble growth and collapse stages than the radius.

A further determination of the bubble growth process could be conducted by calculating the bubble radius changing rate $\dot{r}=d \bar{R} / d t$ and the vapor mass changing rate $\dot{m}=d m_{v} / d t$ for Cases L2 to L4, which are illustrated in Figure 8. 

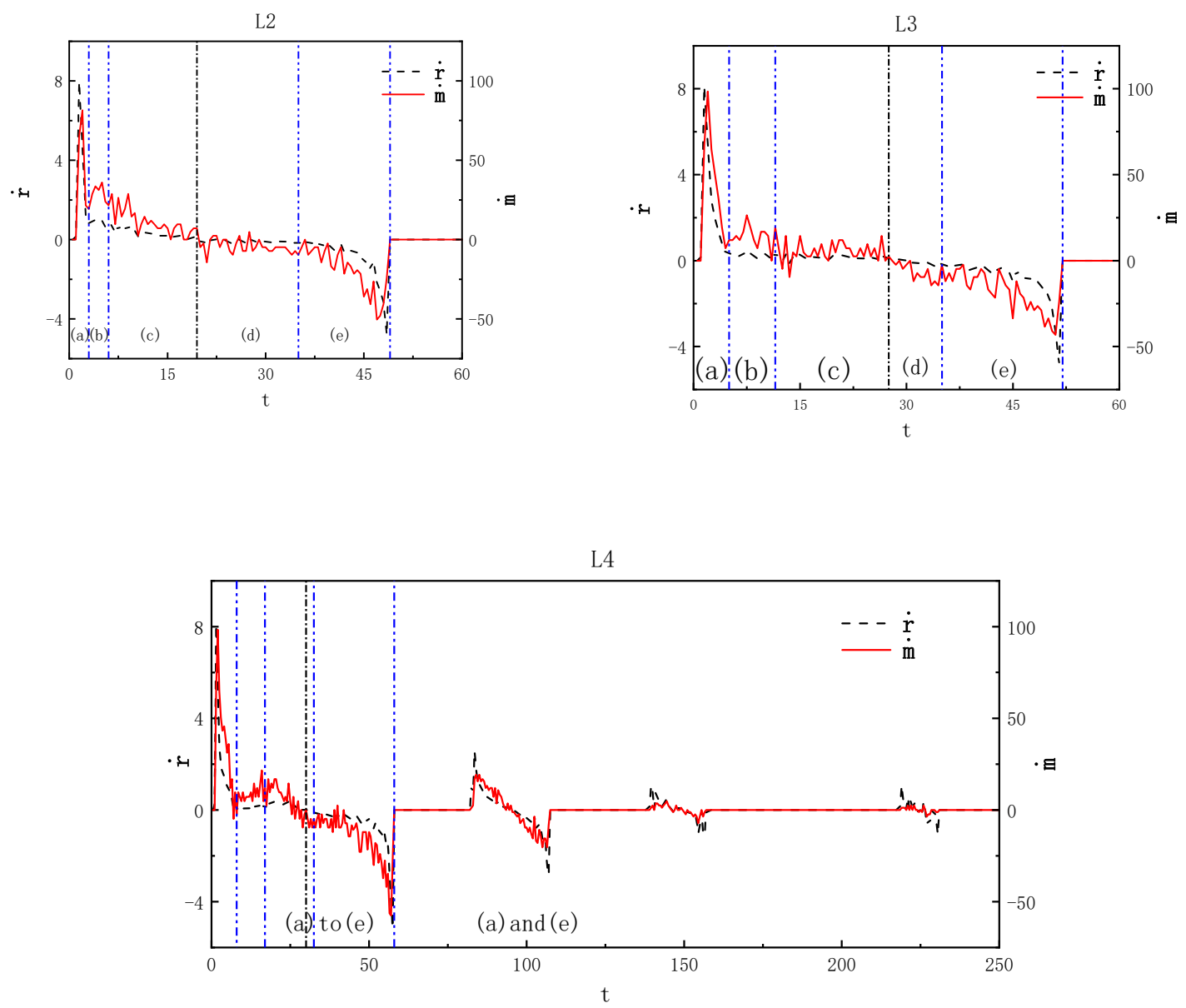

Figure 8. Average radius and vapor mass changing rates of the first bubble for Cases L2 to L4. The short dashed line is the time when the bubble reaches maximum volume. In each case, five bubble stages are identified: (a) inertial-controlled, (b) intermediate, (c) heat-diffusion-controlled, (d) precollapse, and (e) collapse.

Both $\dot{r}$ and $\dot{m}$ reach a peak value in Stage (a). Although the time of the peak is different, $\dot{r}$ changes first. In Stage (b), the magnitude of fluctuation in $\dot{m}$ is marginally larger than that in $\dot{r}$. In Stage (c), $\dot{r}$ and $\dot{m}$ are both at a lower growth rate, but $\dot{r}$ dominates the process. In Stage (d), $\bar{R}$ and $m_{v}$ decrease, with $\dot{r}$ dominating the process. $\dot{m}$ then dominates Stage (e), and $\dot{m}$ changes earlier than $\dot{r}$, especially in the moment of collapse.

As shown in Figure 9, we set a probe into the wall to detect the shockwave released by bubble collapse. With the increase of the heating distance $\gamma$, the impact of the bubble on the wall is weaker. We also find that no secondary bubbles were produced in Cases L2 and L3, but there is still pressure oscillation on the wall. There is a certain threshold for overcoming hydrostatic pressure for bubble formation.

From the above analysis, we could conclude that the pressure force of the expansion wave is the driving force for bubble growth, whereas for bubble shrink, liquefaction occurs earlier than the visible radius contraction. Liquefaction generates huge heat release. When near the wall, as in these cases, the released heat could be transported around the fluid by periodic mass oscillations, albeit in a relatively long time. However, for the bubble at the wall, the impact time is short, and heat transport is limited by the solid wall and the nearly stationary fluid. This could help us understand why a shockwave generates more serious damage when the bubble collapses violently on the wall, which will be discussed in the next section. 


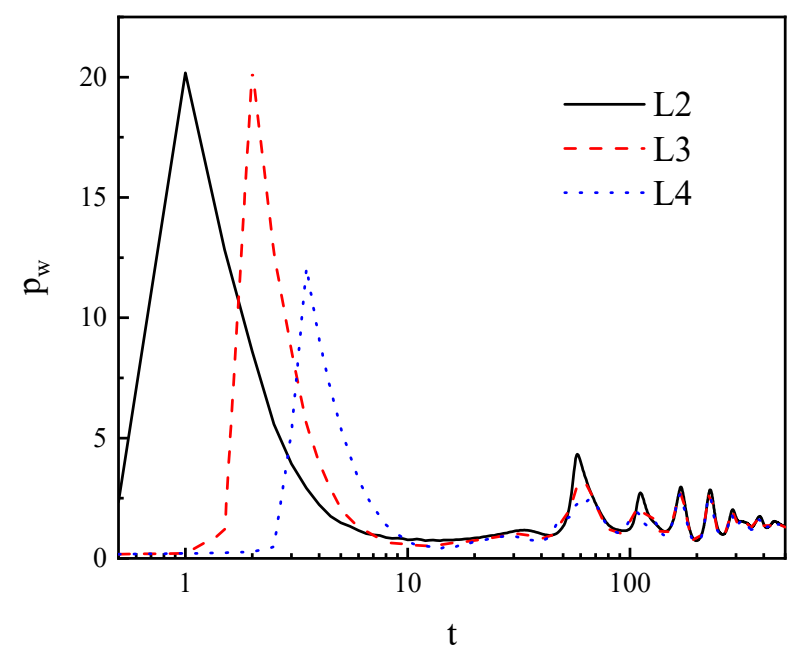

Figure 9. Probe pressure for Cases L2 to L4.

\subsection{Bubble at the Wall}

Six cases, h2, h4, h6, h7, h8, and h10, were set at the solid wall. Here, the heating distance $\mathrm{L}$ was 0 and the liquid heights $\mathrm{h}$ were 20,40,60,70, 80, and 100, respectively, regarding the initial liquid particle spacing distance $\mathrm{dx}$.

Figure 10 shows the bubble density evolution and velocity vector for the different cases. In Case h2, the vapor bubble expands rapidly from the bottom and contacts the free surface. When the bubble exceeds the limit of the free surface, it explodes and splits into multiple parts. Some fluid is quickly splashed to the cold environment, where the hot vapor is quickly liquefied to form many small isolated drops.

In Case h4, the maximum bubble diameter is slightly larger than the thickness of the fluid layer. The bubble bulges the liquid film but does not burst, where a very thin liquid bridge is formed at the top of the bubble. We believe that the surface tension of the liquid film stops the vapor bubble from breaking. Padilla-Martinez observed a similar phenomenon and concluded that the surface tension inhibited the vapor bubble growth when the radius was extremely small and could maintain its relative stability to prevent bursting when the vapor bubble was close to the adjacent radius [10].

In the other cases of a greater liquid height (Cases h6 to h10), the vapor bubble remains under the free surface. The difference is that there is a secondary bubble in Cases h6 and h7 but none in Cases h8 and h10.

In Figure 11, the bubble snapshots of $\eta=1.8$ show that our numerical results are in good agreement with the experimental results of Nguyen [35]. During the growth stage, the bubbles appear to be elongated in the vertical direction. During the collapse stage, the bubbles become flattened. Bubbles then appear as secondary bubbles, and the tip of the secondary bubble is sharp. Due to the effect of the wall, we can observe diverse bubble shapes at the collapse stage in many of the experimental snapshots, being, for example, shell-, flame-, cap-, or droplet-shaped. Although our simulations and experiments show a similar bubble size evolution during growth and collapse, the bubble attachment on the wall is marginally different. This might be caused by the mismatch of the meniscus-covered container used in the experiments vs. the open-topped container in our simulations. Such a meniscus cover was used to suppress the bubble but was difficult to model in the simulation. A further validation might be conducted in the future, either with a model improvement or more precise experiments. 

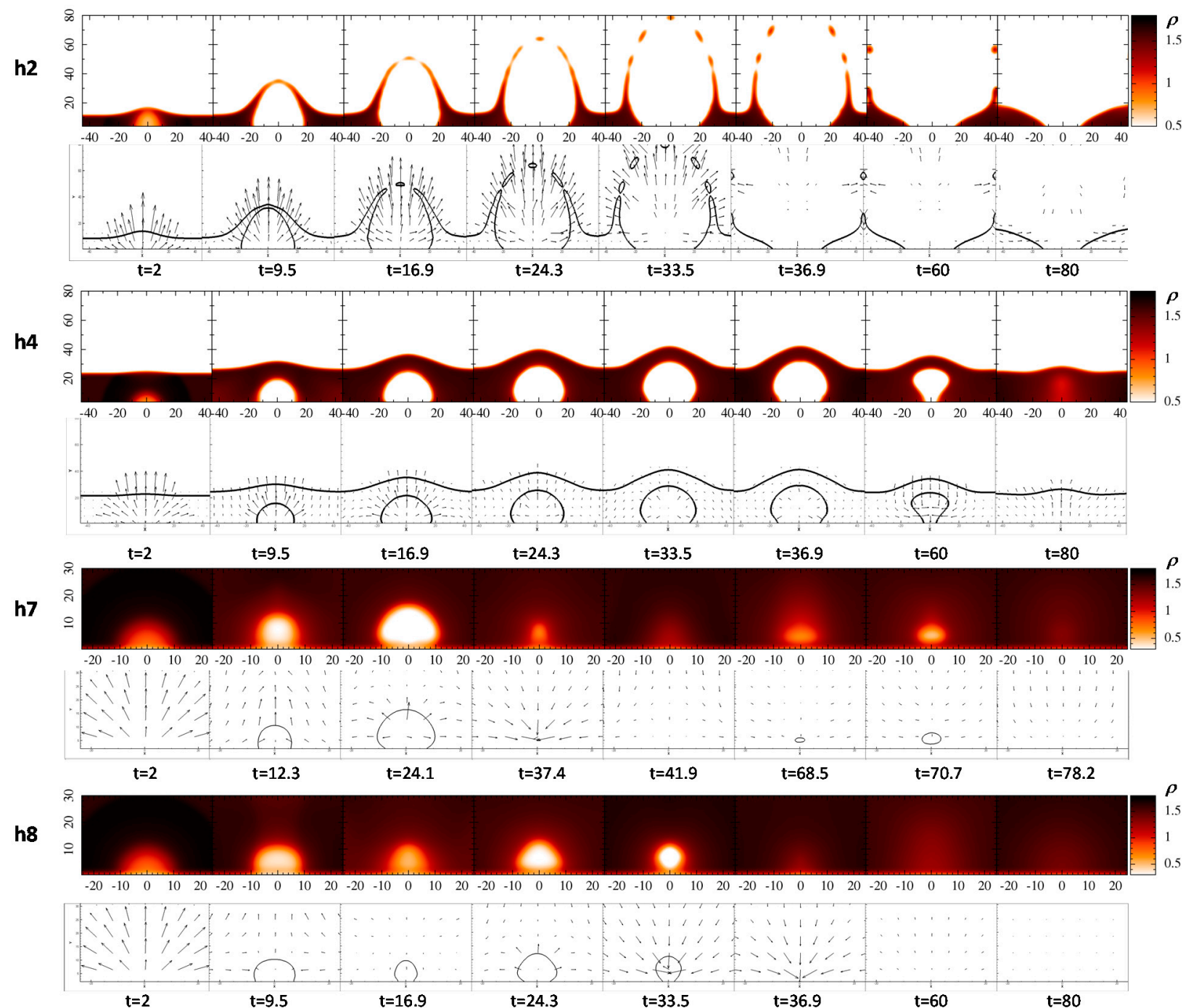

Figure 10. Bubble density counter (top) and flow field vector (below) for Cases h2, h4, h7, and h8.

h6

(a)

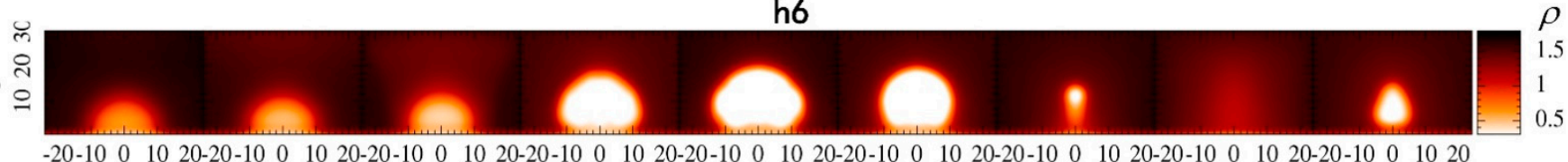

(b)
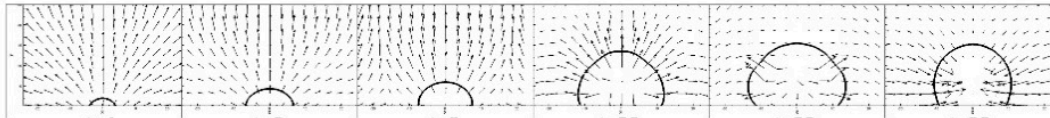

$\mathrm{t}=6$

$\mathrm{t}=8$

$\mathrm{t}=20$

$\mathrm{t}=25$

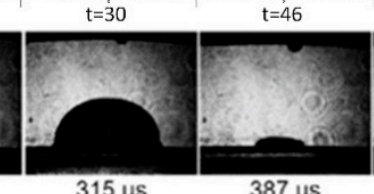

$t=60$

(i)

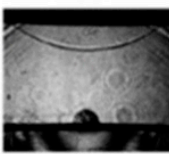

$3 \mu \mathrm{s}$
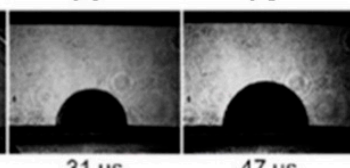

$47 \mu \mathrm{s}$

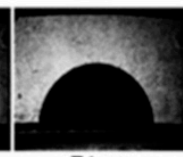

71 us

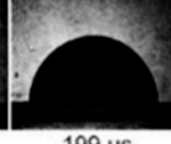

$199 \mu \mathrm{s}$

$315 \mu \mathrm{s}$

$387 \mu \mathrm{s}$
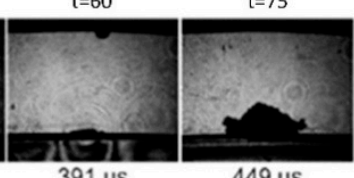

$391 \mu \mathrm{s}$

$449 \mu \mathrm{s}$

Figure 11. (a) Bubble density contour and (b) flow field vector for Case h6 with $\eta=1.8$. (c) Bubble images in the Nguyen experiment with $\mathrm{h}=4 \mathrm{~mm}$, corresponding to $\eta=1.6$ by their maximum bubble radius [35]. The white part in (a) is the vapor phase, and the shadow part in (c) is also the vapor phase.

Figure 12 shows the evolution of the bubble radius $\bar{R}$, bubble average density $\bar{\rho}$, wall pressure $\mathrm{p}_{\mathrm{w}}$, wall temperature $\mathrm{T}_{\mathrm{w}}$, and entropy increase $\Delta S$ with time for Cases $\mathrm{h} 6$, 
$h 7$, and $h 8$. It is found that $p_{w}, T_{w}$, and $\Delta S$ of the bubble have a certain periodicity with the bubble period. It is common that the bubble radius increases at the early stage, following the coincident trajectory. When the bubble growth is close to the maximum value, the bubble behavior is different due to the wall effect. For Cases h6 and h7, Stages (b)-(d) couple together.

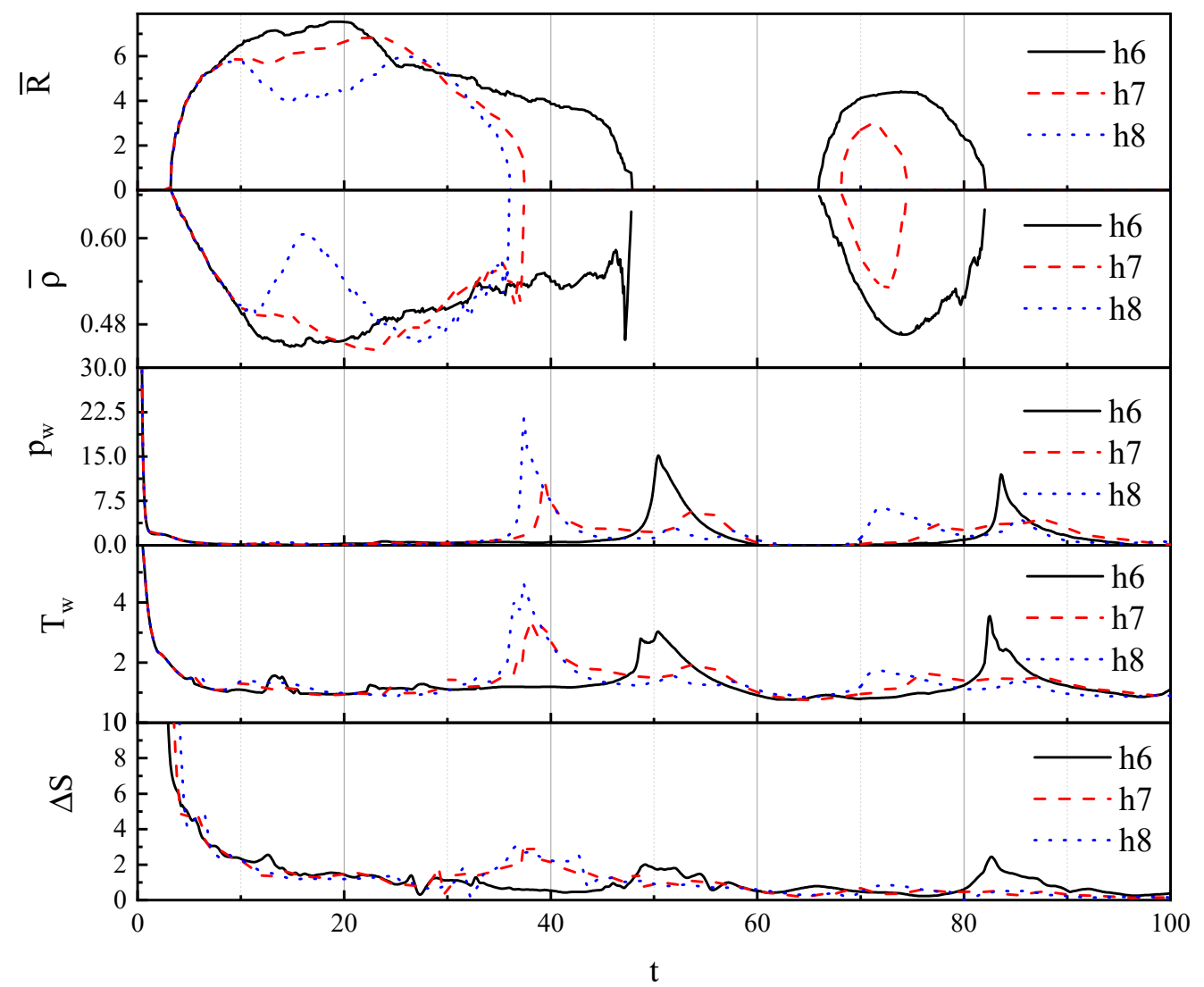

Figure 12. Average bubble radius $\overline{\mathrm{R}}$ and entropy increase $\Delta S$ with time.

After the bubble collapses, the change of $\bar{R}, \Delta S, T_{w}$, and $p_{w}$ shows a certain order. First, it is found that bubble collapse starts with an increase in entropy change. After the bubble collapses, there is a sudden change of $T_{w}$, with a sudden change of $p_{w}$ followed closely. This means that both the fluid pressure and thermal energy increase during collapse. The emergence of the $T_{w}$ peak is even earlier and longer than the $p_{w}$ peak. Although researchers have paid more attention to erosion by pressure, we believe that with an increase in energy, the pressure impact would cause worse erosion damage.

Comparing Cases h6, h7, and h8, no secondary bubble forms with a liquid height greater than 80 due to large hydrostatic pressure. Considering the hydrostatic pressure effect of the heating distance and liquid depth on the vapor bubble, we used $\eta-\gamma$ to estimate the number of secondary bubbles. It can be concluded that if $\eta-\gamma<1$, the bubble bursts through the free surface during its lifetime without secondary bubbles detected. Modestly, if $1<\eta-\gamma<3$, it may see secondary bubbles and more than one emission shockwave after the first bubble collapses. For $3<\eta-\gamma$, there are no secondary bubbles because higher hydrostatic pressure suppresses the appearance of secondary bubbles.

Damage to a wall from bubble collapse is common and important for cavitation and other vapor-bubble-related applications. However, a consensus has not yet been reached for the mechanism of energy conversion during collapse. Zhang et al. believe that the energy of a bubble is transformed into the wave energy of its fluid, causing an impact on a solid wall [24]. Qin found that when a bubble collapses, there is a large amount of heat transfer and energy exchange between the bubble and the outside [23]. 
To illustrate the energy conversion during bubble collapse, we can divide the fluid internal energy into two parts as $E_{U}=E_{h}+E_{u}$, as in Equation (10), with thermal energy $E_{h}=C_{V} T$ and potential energy $E_{u}=-\bar{\alpha} \rho$, respectively. The fluid kinetic energy $E_{k}=\sum_{i=1}^{\text {total }} \frac{1}{2} m_{i} v^{2}$ is also accounted for to represent the mechanical energy information. The transition between these three energy forms is shown in Figure 13. We found that the formation of the bubble is mainly accompanied by thermal fluctuation, which is the mutual conversion of heat energy and potential energy.

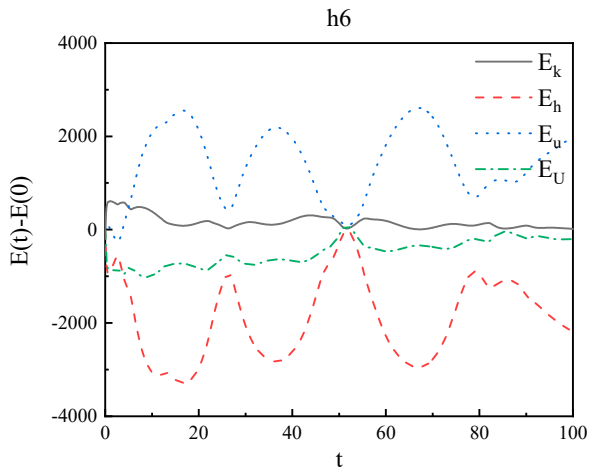

(a)

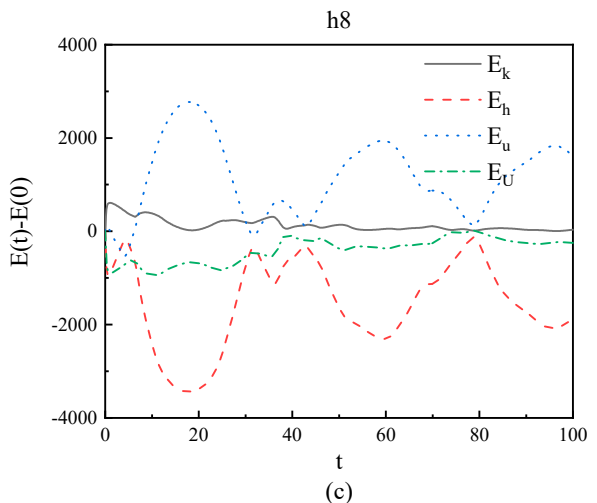

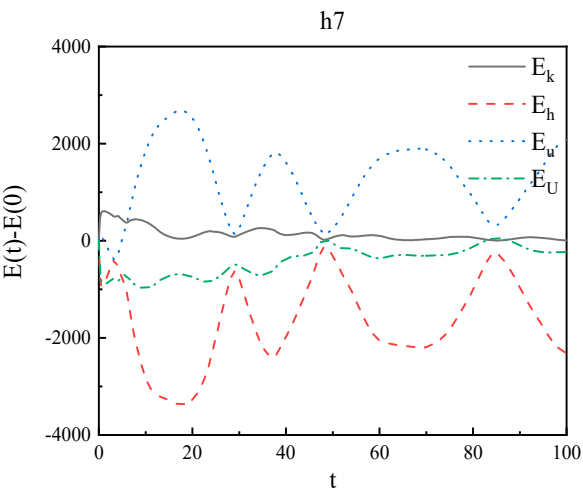

(b)

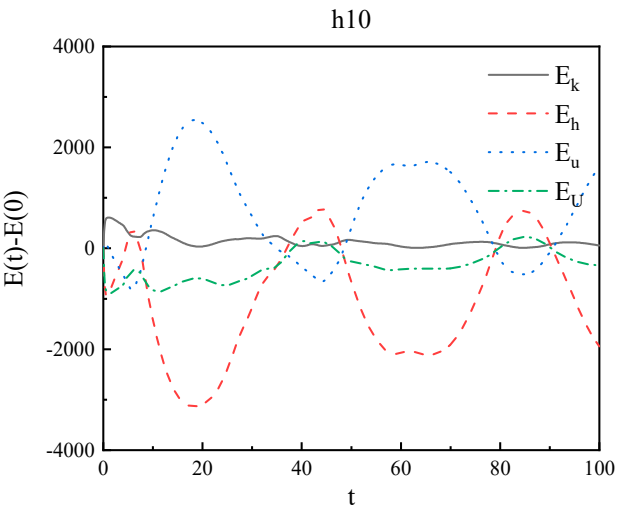

(d)

Figure 13. (a-d) Energy conversion and entropy increase with time for Cases h6 to h10.

Figure 13 clearly reveals an energy conversion relationship at this time. At the moment of collapse completion, $E_{k}$ and $E_{u}$ decrease, but $E_{U}$ and $E_{h}$ increase. It is also observed in Figure 12 that the temperature changes earlier than the pressure changes when the vapor bubble vanishes. We note that the kinetic energy of the bubble collapse is transformed into heat energy. High-temperature fluid in the central area then forms a high-pressure area, which acts as a pressure source to release shockwaves. This high thermal energy causes more damage to the wall beside the pressure impact.

Shockwave pressure causing cavitation erosion on a solid wall is a concerning issue. Zhang showed that during the collapse of a single bubble, the greatest impact on the wall is the $\mathrm{p}_{2 \text { ndmax }}$, which is caused when the bubble is completely collapsed [24]. For laserinduced thermal cavitation bubbles, the initial input energy will also cause extremely highpressure shock $\mathrm{p}_{1 \text { stmax }}$. Calculating the ratio $\mathrm{p}_{2 \text { ndmax }} / \mathrm{p}_{1 \text { stmax }}$ as the shockwave pressure of the secondary bubble collapse over the initial vapor bubble impact pressure, we find $\mathrm{p}_{2 \text { ndmax }} / \mathrm{p}_{1 \text { stmax }}$ in Case $\mathrm{h} 8$ reaches the maximum, as shown in Figure 14 . We conclude that the shockwave impact first increases and decreases at the nondimensional liquid height, with a maximum height of $\eta=4.5$. We define the cavitation potential energy of the impact on the solid wall as $E_{c p}=\int p(t) d t$. In the whole process, $E_{c p}$ reflects the time integral of pressure, and higher statistical pressure will cause more serious cavitation potential. 


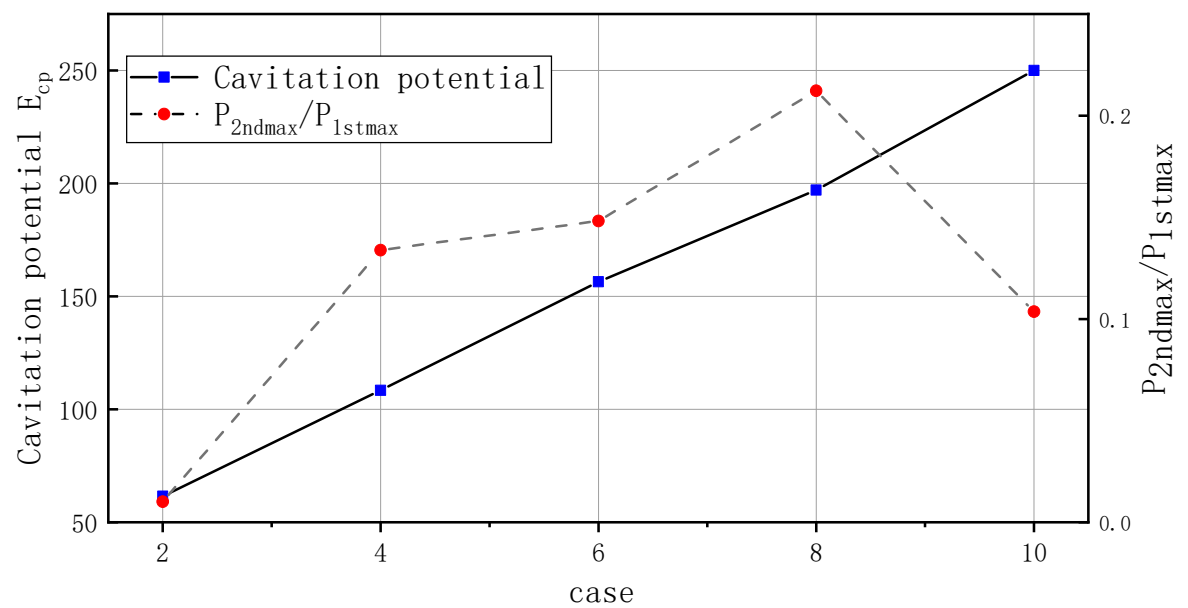

Figure 14. Ratio of two pressure peaks recorded by the probe $\mathrm{p}_{2 n d \max } / \mathrm{p}_{1 \text { stmax }}$ and cavitation potential $\mathrm{E}_{\mathrm{cp}}$ for different cases.

\section{Conclusions}

We used the SPH numerical method to directly simulate bubble growth and collapse processes for a laser-induced bubble near or at a wall.

Bubble radius, density, vapor mass, pressure, temperature, energy, entropy, and their correlations were carefully examined during the bubble's lifetime. The bubble density was found to better describe the bubble behavior than the radius. A new precollapse substage (Stage (d)) was discovered between the growth stages (Stages (a)-(c)) and collapse stage (Stage (e)). The precollapse stage (Stage (d)) was from the point of the largest bubble radius to the next minimum value of bubble density. The mechanism for these five stages was in the out-sync of radius change and mass phase change.

Using this new recognition method based on bubble density, we found there were different substages for bubbles near and at the wall. For the bubble near the wall, the first bubble had five complete stages, from Stages (a) to (e). However, the bubble at the wall only had two clearly defined stages: (a) the inertia-controlled growth stage and (e) the collapse stage. The other three intermittent stages (Stages (b)-(d)) were mixed due to the strong wall effect.

The bubble near the wall had an almost spherical shape, being slightly distorted when floating up slowly. However, the bubble at the wall was nearly hemispherical at growth. When the bubbles collapsed at the wall, they presented diverse shapes, being cap-, shell-, drop-, or flame-shaped. The lifetime of the bubble near the wall was usually longer than that at the wall. Secondary bubbles occurred at a modest hydrostatic pressure of $1<\eta-\gamma<3$ either near or at the wall in the current study. There was a sharp increase in entropy once the bubble completed its collapse, and the kinetic energy of its fluid was converted into heat energy to release the shockwave. Instantaneous erosive damage to the solid wall caused by the shockwave of bubble collapse reached its maximum at nearly $\eta=4.5$.

Author Contributions: Conceptualization, H.W. and H.X.; methodology, C.Z. and H.W.; software, C.Z. and H.W.; validation, H.W.; formal analysis, H.W. and H.X.; investigation, H.W.; resources, H.W.; data curation, H.W.; writing-original draft preparation, H.W.; writing-review and editing, H.X.; visualization, H.W.; supervision, H.X.; project administration, H.W.; funding acquisition, H.X. All authors have read and agreed to the published version of the manuscript.

Funding: This research was funded by the National Natural Science Foundation of China (No. 11972321, No. 91852102).

Data Availability Statement: The data presented in this study are available on request from the corresponding author. The data are not publicly available because of privacy.

Conflicts of Interest: The authors declare no conflict of interest. 


\section{References}

1. Alheshibri, M.; Qian, J.; Jehannin, M.; Craig, V.S.J. A History of Nanobubbles. Langmuir 2016, 32, 11086-11100. [CrossRef] [PubMed]

2. Lin, L.; Peng, X.; Mao, Z.; Li, W.; Yogeesh, M.N.; Rajeeva, B.B.; Perillo, E.P.; Dunn, A.K.; Akinwande, D.; Zheng, Y. Bubble-Pen Lithography. Nano Lett. 2016, 16, 701-708. [CrossRef]

3. Kharangate, C.R.; Mudawar, I. Review of computational studies on boiling and condensation. Int. J. Heat Mass Transf. 2017, 108, 1164-1196. [CrossRef]

4. Jo, H.J.; Kim, S.H.; Park, H.S.; Kim, M.H. Critical heat flux and nucleate boiling on several heterogeneous wetting surfaces: Controlled hydrophobic patterns on a hydrophilic substrate. Int. J. Multiph. Flow 2014, 62, 101-109. [CrossRef]

5. Ekaterina Y, L.; Dmitri O, L. Nanotechnology-Based Platforms. Cancer Nanotechnol. 2016, 1530, $165-192$.

6. Shao, J.; Xuan, M.; Dai, L.; Si, T.; Li, J.; He, Q. Near-infrared-activated nanocalorifiers in microcapsules: Vapor bubble generation for in vivo enhanced cancer therapy. Angew. Chem. Int. Ed. 2015, 54, 12782-12787. [CrossRef] [PubMed]

7. Teirlinck, E.; Fraire, J.; Van Acker, H.; Wille, J.; Swimberghe, R.; Brans, T.; Xiong, R.; Meire, M.; De Moor, R.; De Smedt, S.; et al. Laser-induced vapor nanobubbles improve diffusion in biofilms of antimicrobial agents for wound care. Biofilm 2019, 1, 100004. [CrossRef]

8. Feng, Z.C.; Leal, L.G. Nonlinear Bubble Dynamics. Annu. Rev. Fluid Mech. 1997, 29, 201-243. [CrossRef]

9. Rekhviashvili, S.S. Single-bubble sonoluminescence model. Tech. Phys. Lett. 2008, 34, 1072-1074. [CrossRef]

10. Prosperetti, A. Vapor Bubbles. Annu. Rev. Fluid Mech. 2017, 49, 221-248. [CrossRef]

11. Robinson, P.B.; Blake, J.R.; Kodama, T.; Shima, A.; Tomita, Y. Interaction of cavitation bubbles with a free surface. J. Appl. Phys. 2001, 89, 8225-8237. [CrossRef]

12. Zhang, Y.; Qiu, X.; Zhang, X.; Tang, N. Collapsing dynamics of a laser-induced cavitation bubble near the edge of a rigid wall. Ultrason. Sonochem. 2020, 67, 105157. [CrossRef] [PubMed]

13. Gonzalez-Avila, S.R.; Klaseboer, E.; Khoo, B.C.; Ohl, C.D. Cavitation bubble dynamics in a liquid gap of variable height. J. Fluid Mech. 2011, 682, 241-260. [CrossRef]

14. Sun, C.; Can, E.; Dijkink, R.; Lohse, D.; Prosperetti, A. Growth and collapse of a vapour bubble in a microtube: The role of thermal effects. J. Fluid Mech. 2009, 632, 5-16. [CrossRef]

15. Edel, Z.J.; Mukherjee, A. Experimental investigation of vapor bubble growth during flow boiling in a microchannel. Int. J. Multiph. Flow 2011, 37, 1257-1265. [CrossRef]

16. Kangude, P.; Srivastava, A. Understanding the growth mechanism of single vapor bubble on a hydrophobic surface: Experiments under nucleate pool boiling regime. Int. J. Heat Mass Transf. 2020, 154, 119775. [CrossRef]

17. Lee, H.S.; Merte, H. Spherical vapor bubble growth in uniformly superheated liquids. Int. J. Heat Mass Transf. 1996, 39, 2427-2447. [CrossRef]

18. Rayleigh, L., VIII. On the pressure developed in a liquid during the collapse of a spherical cavity. London, Edinburgh, Dublin Philos. Mag. J. Sci. 1917, 34, 94-98. [CrossRef]

19. Plesset, M.S.; Zwick, S.A. A nonsteady heat diffusion problem with spherical symmetry. J. Appl. Phys. 1952, 23, 95-98. [CrossRef]

20. Ivashnyov, O.E.; Smirnov, N.N. Thermal growth of a vapor bubble moving in superheated liquid. Phys. Fluids 2004, 16, 809-823. [CrossRef]

21. Hao, L. Analysis of bubble growth and motion dynamics in superheated liquid during flash evaporation. Int. J. Heat Mass Transf. 2020, 151, 119356. [CrossRef]

22. Pavlov, P.A. Fast collapse of a vapor bubble. Int. J. Heat Mass Transf. 2017, 104, 627-633. [CrossRef]

23. Qin, Z.; Alehossein, H. Heat transfer during cavitation bubble collapse. Appl. Therm. Eng. 2016, 105, 1067-1075. [CrossRef]

24. Zhang, J.; Zhang, L.; Deng, J. Numerical study of the collapse of multiple bubbles and the energy conversion during bubble collapse. Water 2019, 11, 247. [CrossRef]

25. Wang, Q.; Yao, W.; Quan, X.; Cheng, P. Validation of a dynamic model for vapor bubble growth and collapse under microgravity conditions. Int. Commun. Heat Mass Transf. 2018, 95, 63-73. [CrossRef]

26. Chen, G.Q.; Huang, X.; Zhang, A.M.; Wang, S.P. Simulation of three-dimensional bubble formation and interaction using the high-density-ratio lattice Boltzmann method. Phys. Fluids 2019, 31, 027102.

27. Yang, Y.; Shan, M.; Kan, X.; Shangguan, Y.; Han, Q. Thermodynamic of collapsing cavitation bubble investigated by pseudopotential and thermal MRT-LBM. Ultrason. Sonochem. 2020, 62, 104873. [CrossRef]

28. Wang, Y.; Cai, J. Numerical investigation on bubble evolution during nucleate boiling using diffuse interface method. Int. J. Heat Mass Transf. 2017, 112, 28-38. [CrossRef]

29. Gallo, M.; Magaletti, F.; Cocco, D.; Casciola, C.M. Nucleation and growth dynamics of vapor bubbles. J. Fluid Mech. 2020, 883, 883. [CrossRef]

30. Felderhof, B.U. Dynamics of the diffuse gas-liquid interface near the critical point. Physica 1970, 48, 541-560. [CrossRef]

31. Greiner, W.; Neise, L.; Horst, S. Thermodynamics and Statistical Mechanics; D. Rischke: New York, NY, USA, 1995; pp. 107-110.

32. Yang, X.; Liu, M.; Peng, S. Smoothed particle hydrodynamics modeling of viscous liquid drop without tensile instability. Comput. Fluids 2014, 92, 199-208. [CrossRef]

33. Xiong, H.; Zhang, C.; Yu, Z. Multiphase SPH modeling of water boiling on hydrophilic and hydrophobic surfaces. Int. J. Heat Mass Transf. 2019, 130, 680-692. [CrossRef] 
34. Maheshwari, S.; Van Der Hoef, M.; Prosperetti, A.; Lohse, D. Dynamics of Formation of a Vapor Nanobubble around a Heated Nanoparticle. J. Phys. Chem. C 2018, 122, 20571-20580. [CrossRef] [PubMed]

35. Nguyen, T.T.P.; Tanabe-Yamagishi, R.; Ito, Y. Effects of liquid depth on the expansion and collapse of a hemispherical cavitation bubble induced in nanosecond pulsed laser ablation of a solid in liquid. Opt. Lasers Eng. 2020, 126, 105937. [CrossRef] 